\title{
Crecimiento y mortalidad del pez Haemulon plumieri (Perciformes: Haemulidae) en el archipiélago Los Frailes, Venezuela
}

\author{
Delvalle González ${ }^{1}$, Nora Eslava ${ }^{2 *}$, Leo Walter González² \& Francisco Guevara² \\ 1. Universidad de Oriente. Escuela de Ciencias Aplicadas del Mar. Departamento de Acuacultura. Boca del Río, isla de \\ Margarita, Venezuela; delvalle_mgs05@hotmail.com \\ 2. Universidad de Oriente. Instituto de Investigaciones Científicas. Área de Biología y Recursos Pesqueros. Boca del \\ Río, Isla de Margarita, Venezuela; neslava20@yahoo.es, 1wgc25@gmail.com, franciscogm2002e@hotmail.com \\ * Correspondencia
}

Recibido 21-XII-2018. Corregido 18-X-2019. Aceptado 21-X-2019.

\begin{abstract}
Growth and mortality of Haemulon plumieri fish (Perciformes: Haemulidae) in the Los Frailes Archipelago, Venezuela. The white grunt Haemulon plumieri is abundant in northeastern Venezuela, but the biological and fishery aspects of this species have been little studied in the country. The objective of this study was to estimate the growth and mortality of this resource. We collected samples monthly from January to December 2014, obtaining 2967 specimens. Total length $(\mathrm{cm})$ and weight $(\mathrm{g})$ were determined for each specimen. No sexual dimorphism was shown with respect to length $\left(\mathrm{t}_{\mathrm{S}}=0.093 ; \mathrm{P}>0.05\right)$. Thus, one length-weight ratio was established for both sexes: $\mathrm{W}=1.4 \times 10^{-3 *} \mathrm{~L}^{2.99}$, which showed isometric growth. FiSAT package routines were used to estimate the growth parameters of the individual growth model of von Bertalanffy: $\mathrm{L}_{\infty}=$ $30.95 \mathrm{~cm}, \mathrm{k}=0.67 /$ year, $\mathrm{W}_{\infty}=418.17 \mathrm{~g}, \mathrm{t}_{0}=-0.24$ years. We estimated a max age of 4 years. The coefficient of variation of the growth performance index $\left(\varnothing^{\prime}\right)$ for the direct method $(\mathrm{CV}=5.26 \%)$ showed a variability in growth patterns according to region. The rate of natural mortality $(\mathrm{M}=1.36 /$ year $)$ was high, possibly due to predation and disease, among others. Rates of fishing mortality $(\mathrm{F}=1.95 /$ year $)$ and total mortality $(\mathrm{Z}=3.31$ / year) were high. The exploitation rate $(\mathrm{E}=0.59 /$ year $)$ indicates that $H$. plumieri is likely overexploited.
\end{abstract}

Key words: Haemulon plumieri, growth, mortality, Los Frailes Archipelago, Venezuela.

González, D., Eslava, N., González, L. W., \& Guevara, F. (2019). Crecimiento y mortalidad de Haemulon plumieri (Perciformes: Haemulidae) en el archipiélago Los Frailes, Venezuela. Revista de Biología Tropical, 67(6), 1560-1571.

Haemulon plumieri se encuentra distribuida desde el sureste de Estados Unidos hasta Brasil, incluyendo el Mar Caribe y el Golfo de México. En Venezuela es capturada por la pesca artesanal principalmente con nasa, cordel y red. Habita en fondos someros, hasta $\sim 40$ $\mathrm{m}$ de profundidad con formaciones rocosas o coralinas, pero no asociados a ellas. Los ejemplares pequeños de 7 a $9.5 \mathrm{~cm}$ de longitud total se capturan en praderas de Thalassia (Cervigón, 1993). H. plumieri es una especie carnívora y los adultos se alimentan principalmente de invertebrados bentónicos, como crustáceos y poliquetos (Manooch, 1976). Es un recurso importante en las pesquerías artesanales del estado Nueva Esparta, e.g., la flota artesanal de El Tirano que faena en el archipiélago Los Frailes desembarcó 35.6 toneladas en la temporada de pesca 2012 representando el $4.11 \%$ de las 51 especies de peces capturados (González, Eslava, Guevara, Díaz, \& Rodríguez, 2017).

En el contexto de las pesquerías multiespecíficas no es fácil aplicar dispositivos de gestión y regulación pesquera, aun reconociendo 
su significación en la sostenibilidad biológica, económica y social. Sin embargo, es necesario realizar estudios de dinámica poblacional con la finalidad de evaluar los recursos, considerando los parámetros de crecimiento, reclutamiento y mortalidad, teniendo en cuenta que el número de individuos en cada clase anual va disminuyendo debido a la mortalidad natural o por pesca, siendo este último parámetro utilizado en la administración pesquera. Básicamente, hay una fracción de individuos que mueren por diversas causas y otra que no mueren y llegan al final del ciclo de vida (Csirke, 1980).

Se han realizado diversos estudios biológico-pesqueros de $H$. plumieri, principalmente en Norte América. Así, Manooch (1976), determinó la edad, crecimiento y mortalidad, Darcy (1983) realizó una sinopsis de su biología, Murphy, Murie y Muller (1999) y Potts (2000) describieron las características biológico-pesqueras, y estimaron la estructura de edad y tamaño de la población a través del análisis de población virtual (APV), mortalidad por pesca, mortalidad natural y reclutamiento. Silva y Murphy (2001) realizaron un resumen de su biología y pesquería en la costa este de Florida; Potts y Manooch (2001) y Murie y Parkyn (2005) estimaron la edad y crecimiento en Carolina y Florida, respectivamente. Por otra parte, en Brasil, Nomura (1965) analizó la relación longitud-peso, Araújo y Martins (2007) estudiaron edad, crecimiento y mortalidad; Mota y Aragao (1973) y Shinozaki-Mendes, Santander, Silva y Hazin (2013a) establecieron la madurez sexual mediante características macro y microscópica (ovogénesis y espermatogénesis) y tipo de desove; y ShinozakiMendes, Santander, Silva y Hazin (2013b) investigaron la variación estacional de las etapas de maduración gonadal, talla de primera madurez sexual $\left(\mathrm{L}_{50}\right)$ y fecundidad. Así mismo, en Jamaica Billings y Munro (1974) estudiaron la biología, ecología y bionomía; en Puerto Rico, Sadovy, Figuerola y Román (1989) y en Antigua, Constantine (2008) examinaron la edad y crecimiento; en Yucatán, DomínguezViveros y Ávila-Martínez (1986) estudiaron la pesquería. Finalmente, Treinen-Crespo,
Villegas-Hernández, Guillén-Hernández, RuizZárate y González-Salas (2012) realizaron un análisis de la forma del otolito como herramienta para la diferenciación poblacional. Es importante señalar que en Venezuela se han realizado estudios sobre la descripción taxonómica de la especie (Cervigón, 1993) y la proporción sexual, talla mínima de madurez, época de reproducción y fecundidad (PalazónFernández, 2007). Debido al desconocimiento de la dinámica poblacional de $H$. plumieri, sobre todo en los actuales momentos en que se ha convertido en un recurso de alta demanda para el consumo por su carne de excelente calidad (Cervigón, 1993), se determinó estimar su crecimiento y mortalidad, para contribuir a la evaluación y correcto manejo pesquero.

\section{MATERIALES Y MÉTODOS}

Recolecta de muestras: Los ejemplares de corocoro margariteño Haemulon plumieri fueron obtenidos de las capturas comerciales de la flota artesanal de El Tirano que utilizan nasa hexagonal tipo antillano elaborada con malla de plástico y alambre galvanizado de $5 \mathrm{~cm}$ de abertura, sin carnada colocadas en "enyugue" (grupo de tres o más unidas por una cuerda y separadas a una distancia de 15 a $20 \mathrm{~m}$ ) sobre el fondo marino (González, Eslava, \& Guevara, 2006) a una profundidad aproximada de 20 a 40 $\mathrm{m}$. Las faenas de pesca se efectuaron en zonas cercanas al archipiélago Los Frailes, ubicado al noreste de la isla de Margarita, estado Nueva Esparta (11 ${ }^{\circ} 11^{\prime} 30^{\prime \prime} \mathrm{N} \& 63^{\circ} 42^{\prime} 00^{\prime}$ 'W) (Fig. 1).

Los muestreos se realizaron semanalmente de enero a diciembre 2014 en el puerto de El Tirano. El número de individuos muestreados mensualmente fluctuó entre 177 y 360 . A cada ejemplar se determinó su longitud total (L) en $\mathrm{cm}$, comprendida desde extremo anterior de la boca hasta el extremo posterior de los radios más largos de la aleta caudal, utilizando un ictiómetro marca Wildco modelo 118 con apreciación de $1 \mathrm{~mm}$, y el peso total sin eviscerar (P) en g mediante una balanza electrónica marca Sartorius modelo 1106 con precisión de $0.01 \mathrm{~g}$ y $1 \mathrm{~kg}$ de capacidad máxima. La 


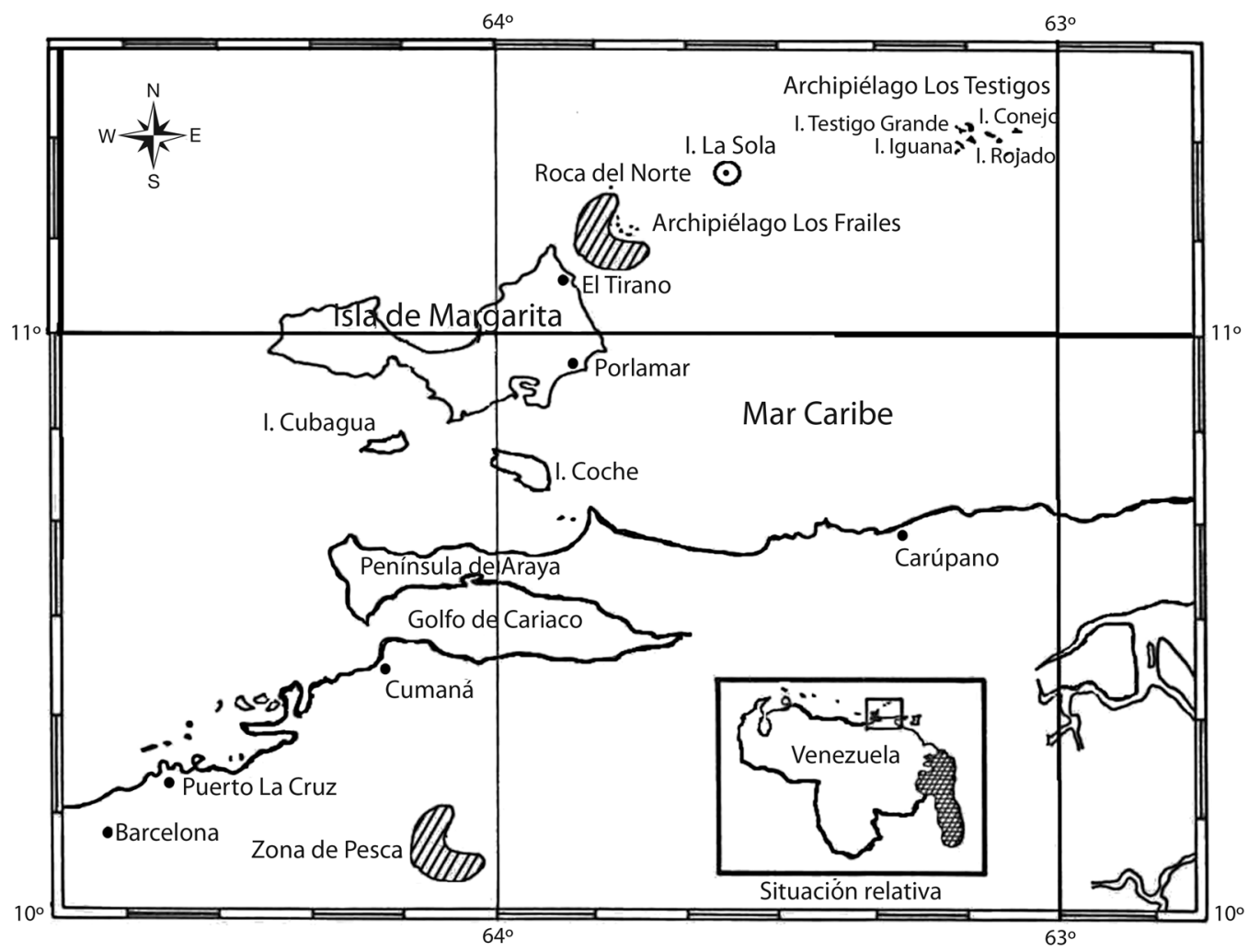

Fig. 1. Zona de pesca del archipiélago Los Frailes, Venezuela. Modificado de González, Eslava y Guevara (2001).

Fig. 1. Fishing zone from Los Frailes Archipelago, Venezuela. Modified by González, Eslava y Guevara (2001).

identificación sexual se estableció mediante la observación de las gónadas, según PalazónFernández (2007).

Análisis estadístico: Las diferencias estadísticas significativas entre las longitudes por sexo se precisaron con la aplicación de la prueba t-student (Zar, 2009). La relación entre longitud y peso se determinó mediante la ecuación potencial de Ricker (1975): $\mathrm{P}=a^{*} \mathrm{~L}^{b}$; donde $\mathrm{P}$ es el peso total $(\mathrm{g})$, L es la longitud total $(\mathrm{cm}), a$ es la intersección con el eje y, $b$ es la pendiente de la curva que indica la isometría o alometría en el crecimiento. Las constantes $a$ y $b$ fueron estimadas por el método de mínimos cuadrados, previa linealización de los datos mediante una transformación logarítmica (ln). Se aplicó la hipótesis del crecimiento isométrico (Ricker, 1975) mediante la prueba t-student (Walpole,
Myers, Myers, \& Ye, 2007) con un nivel de significación de $\alpha=0.05$.

Parámetros poblacionales: En la estimación de los parámetros poblacionales se utilizaron las longitudes comprendidas entre 14 y $31 \mathrm{~cm}$, debido a que las tallas de 32 a 41 $\mathrm{cm}$ estuvieron poco representadas (21 ejemplares). El cálculo de la longitud asintótica $\left(\mathrm{L}_{\infty}\right)$ y el coeficiente de crecimiento o curvatura $(\mathrm{k})$ de la ecuación de crecimiento de von Bertalanffy (1938), se basó en el método indirecto del análisis de la distribución mensual de las frecuencias de longitud de $H$. plumieri con un rango de $1 \mathrm{~cm}$, utilizando las rutinas del paquete FiSAT (FAO-ICLARM Stock Assessment Tools) (Gayanilo, Sparre, \& Pauly, 1996). Se consideró una longitud asintótica preliminar aplicando el Método de Powell (1979) y 
Wetherall (1986), que permitió determinar el coeficiente de crecimiento mediante la rutina ELEFAN I (Pauly \& David, 1981), previa reestructuración de las frecuencias de longitud. Para la optimización de los parámetros de $\mathrm{L}_{\infty}$ y k se empleó el procedimiento de Gulland y Holt (1959) asociado a la misma rutina, previo análisis de la progresión modal utilizando la descomposición de la frecuencia de las distribuciones mensuales de longitud según el método de Bhattacharya (1967). Una vez determinadas las medias de los grupos modales por mes, estas se unieron entre meses para obtener los datos del incremento de crecimiento. Se calculó el $\mathrm{t}_{\mathrm{o}}$ de acuerdo a la fórmula indicada por Pauly (1979): $\log _{10}\left(-t_{\mathrm{o}}\right)=-0.3922-0.2752$ $\log _{10} \mathrm{~L}_{\infty}-1.038 \log _{10} \mathrm{k}$; donde $\mathrm{t}_{\mathrm{o}}$ es la edad teórica en que el organismo tiene talla cero, $\mathrm{L}_{\infty}$ es la longitud asintótica o longitud máxima promedio y $\mathrm{k}$ es el coeficiente de crecimiento o coeficiente de curvatura.

El peso asintótico se estimó mediante la fórmula de Csirke (1980): $\mathrm{P}_{\infty}=a^{*} \mathrm{~L}_{\infty}{ }^{b}$; donde $\mathrm{P}_{\infty}$ es el peso asintótico o peso máximo promedio. Para el cálculo de la longevidad se utilizó la ecuación de Taylor (1958): $\mathrm{A}_{0.95}=\mathrm{t}_{\mathrm{o}}+2.996$ / $\mathrm{k}$ donde $\mathrm{A}_{0.95}$ es la edad máxima o tiempo requerido para alcanzar el $95 \%$ de la longitud máxima del pez $\left(\mathrm{L}_{\infty}\right)$. Una vez calculados los parámetros de la ECVB $\left(\mathrm{L}_{\infty}, \mathrm{k} \mathrm{y} \mathrm{t}_{\mathrm{o}}\right)$, se establecieron las curvas de crecimiento ajustadas al modelo de von Bertalanffy (1938) en longitud y peso respectivamente, hasta la edad límite, según las siguientes ecuaciones: $\mathrm{L}=\mathrm{L}_{\infty}\left[1-\mathrm{e}^{-\mathrm{k}}\right.$ ( $\mathrm{t}-\mathrm{to})] ; \mathrm{P}=\mathrm{P}_{\infty}\left[1-\mathrm{e}^{-\mathrm{k}(\mathrm{t}-\mathrm{to})}\right]^{\mathrm{b}}$. Se determinó el índice de desempeño del crecimiento phi prima $\left(\varnothing^{\prime}\right)$ para comparar los parámetros de crecimiento con los resultados determinados por otros investigadores en diferentes regiones, de acuerdo a la fórmula de Munro y Pauly (1983): $\varnothing^{\prime}=\log _{10} \mathrm{k}+2 \log _{10} \mathrm{~L}_{\infty}$. Se consideró el criterio de Sparre y Venema (1997), quienes señalaron que el coeficiente de variación $(\mathrm{CV})$ de $\varnothing^{\prime}$ no debe exceder de $4 \%$ para asumir que los resultados son estadísticamente similares.

La mortalidad natural (M) se calculó utilizando la ecuación empírica de Pauly (1980): $\log _{10}(\mathrm{M})=-0.0066-0.279 \log _{10} \mathrm{~L}_{\infty}+0.6543$ $\log _{10} \mathrm{k}+0.4634 \log _{10} \mathrm{~T}$ con la temperatura superficial media del agua de $28^{\circ} \mathrm{C}$. La mortalidad total $(Z)$ se calculó mediante el método de la curva de captura linealizada basada en datos de longitud (Pauly, 1983). La mortalidad por pesca (F) se calculó de la diferencia entre $Z$ y $\mathrm{M}$, y la tasa de explotación (E) según Gulland (1971): $\mathrm{E}=\mathrm{F} / \mathrm{Z}$ quien indicó que la tasa de explotación óptima (Eóptima) ocurre cuando $\mathrm{E}=0.5 /$ año

\section{RESULTADOS}

Se examinó un total de 2967 ejemplares (2 084 juveniles y 883 adultos) con longitudes entre 14 y $41 \mathrm{~cm}$ (Media $=20.58 \mathrm{~cm} \mathrm{DE} \pm$ 2.42) y pesos que fluctuaron entre 45 y 890 $\mathrm{g}($ Media $=131.22 \mathrm{~g} \mathrm{DE} \pm 60.23)$. El mayor número de individuos se registró en agosto, mientras que el menor en diciembre. La menor longitud se encontró en enero y la mayor en octubre (Tabla 1). No se encontraron diferencias estadísticamente significativas para la longitud entre hembras y machos $\left(\mathrm{t}_{\mathrm{s}}=0.093 ; \mathrm{P}\right.$ $>0.05$ ), por lo que se determinó el crecimiento y la mortalidad para los sexos combinados. En los meses estudiados, excepto febrero, $H$. plumieri presentó la mayor frecuencia de longitud entre 18 y $23 \mathrm{~cm}$, siendo la moda de $20 \mathrm{~cm}$.

\section{Crecimiento}

La relación longitud-peso quedó establecida como $\mathrm{P}=1.4 \times 10^{-3} * \mathrm{~L}^{2.99}$ siendo la constante $b$ igual a $3\left(\mathrm{t}_{\mathrm{s}}=-0.302 ; \mathrm{P}>0.05\right)$, demostrando así un crecimiento isométrico (Fig. 2), lo que indica que los ejemplares, tienen un crecimiento individual donde las proporciones geométricas del cuerpo no cambian (crecimiento homogéneo).

Los grupos modales por mes no estuvieron bien definidos. Se realizó una primera estimación de la longitud asintótica $\left(\mathrm{L}_{\infty}\right)$ igual a 30.95 cm (Fig. 3A), que fue utilizada como dato de entrada en la rutina ELEFAN I para la estimación de k. Luego de varias corridas, se obtuvo una primera valoración de $\mathrm{k}=0.67 / \mathrm{año}$, evidenciándose el inicio de la curva de crecimiento 
TABLA 1

Ejemplares recolectados de Haemulon plumieri, enero-diciembre 2014 en el archipiélago Los Frailes, Venezuela

TABLE 1

Haemulon plumieri specimens collected during January-December 2014 from Los Frailes Archipelago, Venezuela

\begin{tabular}{lccccccc}
\multicolumn{1}{c}{ Mes } & $n$ & \multicolumn{3}{c}{ Longitud total $(\mathrm{cm})$} & \multicolumn{3}{c}{ Peso total $(\mathrm{g})$} \\
Enero & 241 & $14-38$ & 20.30 & 2.39 & $48-801$ & 126.71 & 61.65 \\
Febrero & 252 & $16-37$ & 19.87 & 4.53 & $45-800$ & 135.59 & 116.62 \\
Marzo & 273 & $16-31$ & 19.11 & 1.94 & $57-493$ & 101.9 & 41.50 \\
Abril & 234 & $17-26$ & 19.33 & 1.29 & $58-240$ & 100.78 & 21.98 \\
Mayo & 335 & $16-31$ & 20.22 & 2.08 & $60-468$ & 123.96 & 50.58 \\
Junio & 204 & $16-27$ & 20.62 & 1.56 & $60-332$ & 131.26 & 30.94 \\
Julio & 206 & $17-35$ & 20.34 & 2.21 & $69-639$ & 130.48 & 55.93 \\
Agosto & 360 & $17-32$ & 21.17 & 2.12 & $67-343$ & 138.49 & 43.66 \\
Septiembre & 249 & $16-26$ & 20.13 & 1.76 & $58-244$ & 115.27 & 34.66 \\
Octubre & 205 & $16-41$ & 21.94 & 3.86 & $72-890$ & 161.33 & 127.72 \\
Noviembre & 231 & $16-33$ & 22.28 & 2.43 & $61-499$ & 159.15 & 65.44 \\
Diciembre & 177 & $16-33$ & 21.68 & 2.91 & $64-528$ & 149.78 & 72.05 \\
Total & 2967 & & 20.58 & 2.42 & & 131.22 & 60.23 \\
\hline
\end{tabular}

$n=$ número ejemplares examinados; Min = valor mínimo; Max = valor máximo; Media = valor medio;

$\mathrm{DE}=$ desviación estándar.

$n=$ number of specimens examined; Min = minimum value; Max = maximum value; Media $=$ mean value; $\mathrm{DE}=$ standard deviation.

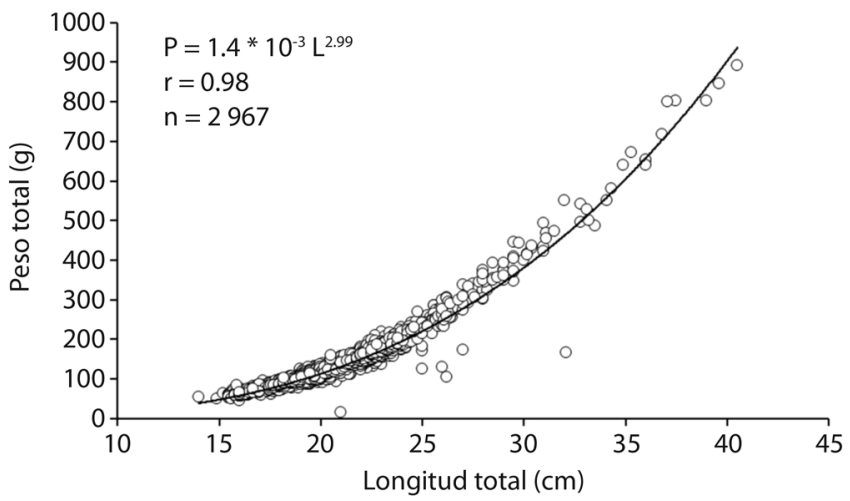

Fig. 2. Relación longitud total - peso total de Haemulon plumieri del archipiélago Los Frailes, Venezuela de enero a diciembre 2014.

Fig. 2. Total length - total weight relationship of Haemulon plumieri from Los Frailes Archipelago, Venezuela between January and December 2014.

en longitud en mayo. La bondad de ajuste entre la $\mathrm{L}_{\infty} \mathrm{y}$ el $\mathrm{k}$ fue de $\mathrm{Rn}=0.271$. Posteriormente se aplicó la rutina de Gulland y Holt (1959), la cual proporcionó valores adicionales de $\mathrm{L}_{\infty} \mathrm{y} \mathrm{k}$, los que fueron descartados por no corresponder a las características biológicas de la especie
(Fig. 3B). Se estimó el $t_{\mathrm{o}}=-0.24$ años, lo que permitió finalmente determinar la edad límite $\mathrm{A}_{0.95}=4$ años. Se obtuvo un peso asintótico de $418.17 \mathrm{~g}$, utilizando las constantes $a=1.4 \times 10^{-}$ 3 y $b=2.99$ de la ecuación de la relación 

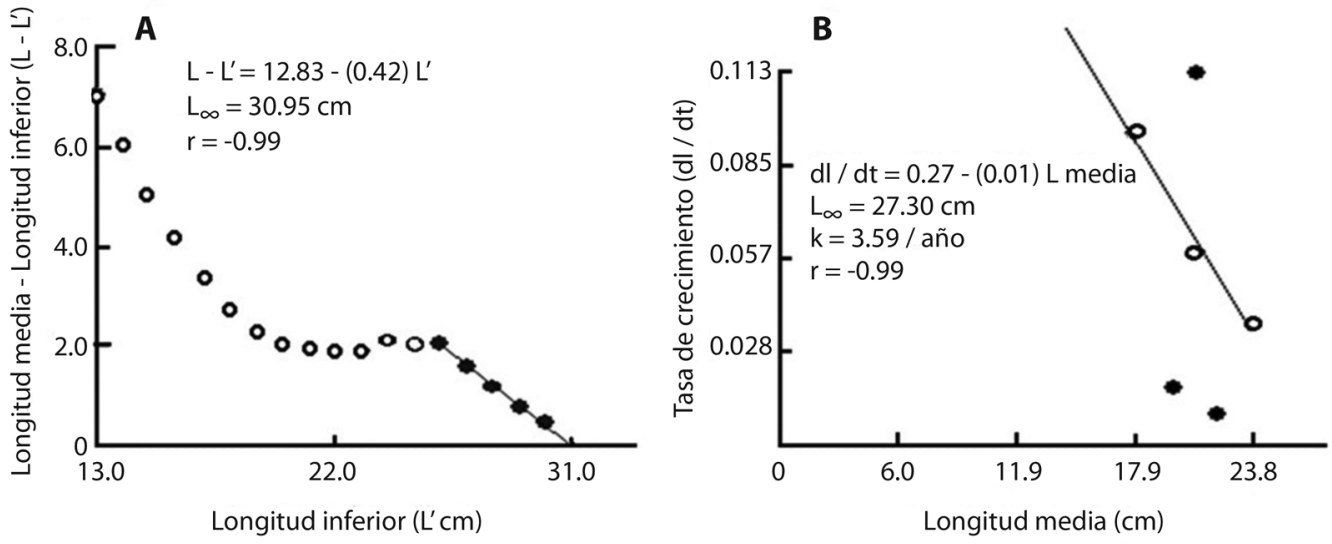

Fig. 3. A. Estimación de la longitud asintótica $\left(\mathrm{L}_{\infty}\right)$ aplicando el modelo de Powell (1979) y Wetherall (1986). B. Estimación de la longitud asintótica $\left(\mathrm{L}_{\infty}\right)$ y el coeficiente de crecimiento (k) aplicando el modelo de Gulland \& Holt (1959) de Haemulon plumieri del archipiélago Los Frailes, Venezuela, enero-diciembre 2014. Valores considerados en la regresión lineal en A 1 $\mathrm{y} i$ en $\mathrm{B}$.

Fig. 3. A. Estimation of Asymptotic length $\left(\mathrm{L}_{\infty}\right.$ applying the Powell (1979) and Wetherall (1986) model. B. Estimation of Asymptotic length $\left(\mathrm{L}_{\infty}\right)$ and growth coefficient kapplying the Gulland \& Holt (1959) model of Haemulon plumieri from Los Frailes Archipelago, Venezuela between January and December 2014. Values considered in the linear regression in A 1 and $;$ in $\mathrm{B}$.
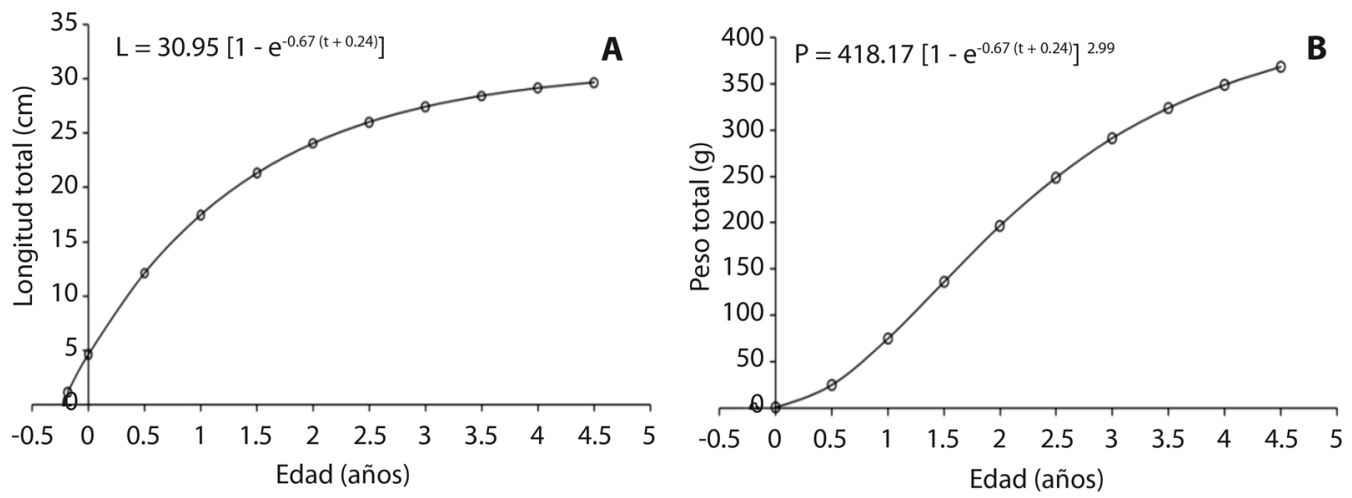

Fig. 4. A. Curva de crecimiento en longitud de Haemulon plumieri según el modelo de von Bertalanffy (1938). B. Curva de crecimiento en peso de Haemulon plumieri según el modelo de von Bertalanffy (1938) del archipiélago Los Frailes, Venezuela de enero a diciembre 2014.

Fig. 4. A. Growth curve in length of Haemulon plumieri, according to the von Bertalanffy (1938) model. B. Growth curve in weight of Haemulon plumieri, according to the von Bertalanffy (1938) model from Los Frailes Archipelago, Venezuela between January and December 2014.

longitud-peso y el parámetro preliminar de crecimiento $\mathrm{L}_{\infty}=30.95 \mathrm{~cm}$.

La curva de crecimiento en longitud describió una curva de tipo exponencial, acelerado en el primer año de vida y luego disminuyó gradualmente hasta alcanzar la longitud máxima teórica (Fig. 4A). La curva de crecimiento en peso mostró un comportamiento sigmoideo lento en el primer año de vida, luego en forma exponencial hasta los dos años y posteriormente, una disminución paulatina hasta alcanzar su peso asintótico (Fig. 4B). El coeficiente 
TABLA 2

Parámetros de crecimiento de Haemulon plumieri estimados por diferentes autores

TABLE 2

Growth parameters of Haemulon plumieri estimated by different authors

\begin{tabular}{|c|c|c|c|c|c|c|}
\hline Autor & Región & Sexo & $\mathrm{L}_{\infty}(\mathrm{cm})$ & k (año) & $\varnothing^{\prime}$ & $\mathrm{A}_{0.95}$ (años) \\
\hline \multicolumn{7}{|l|}{ Método directo (otolitos) } \\
\hline \multirow[t]{2}{*}{ Potts \& Manooch (2001) } & Sureste de Florida (EUA) & Combinados & 32.70 & 0.19 & 2.30 & 15 \\
\hline & Norte y sur de Carolina (EUA) & Combinados & 59.10 & 0.08 & 2.44 & 13 \\
\hline \multirow[t]{5}{*}{ Murie \& Parkyn (2005) } & Costa del Golfo de Florida (EUA) & & & & & \\
\hline & Norte-Central & Hembras & 31.70 & 0.35 & 2.54 & 18 \\
\hline & & Machos & 34.50 & 0.41 & 2.68 & \\
\hline & Central & Hembras & 29.50 & 0.30 & 2.41 & \\
\hline & & Machos & 31.90 & 0.36 & 2.56 & \\
\hline \multirow[t]{3}{*}{ Araújo \& Martins (2007) } & Costa Central de Brasil (Brasil) & Combinados & 31.22 & 0.48 & 2.67 & 28 \\
\hline & & Hembras & 29.80 & 0.59 & 2.71 & \\
\hline & & Machos & 33.10 & 0.35 & 2.58 & \\
\hline \multirow[t]{2}{*}{ Constastine (2008) } & Antigua (Costa sur oriental) & Combinados & 28.20 & 0.36 & 2.45 & 14 \\
\hline & & Coeficiente $\mathrm{d}$ & le Variació & $n(C V)$ & 5.26 & \\
\hline \multicolumn{7}{|c|}{ Método indirecto (frecuencia de tallas) } \\
\hline Billings \& Munro (1974) & Arrecife de Port Royal (Jamaica) & Combinados & 42.00 & 0.37 & 2.81 & 8 \\
\hline $\begin{array}{l}\text { Domínguez-Viveros } \\
\text { \& Ávila-Martínez (1986) }\end{array}$ & Banco de Campeche (México) & Combinados & 45.43 & 0.29 & 2.78 & 11 \\
\hline \multirow[t]{2}{*}{ Este estudio } & Archipiélago Los Frailes (Venezuela) & Combinados & 30.95 & 0.67 & 2.80 & 4 \\
\hline & & \multicolumn{3}{|c|}{ Coeficiente de Variación (CV) } & 0.55 & \\
\hline
\end{tabular}

$\mathrm{L}_{\infty}=$ longitud asintótica; $\mathrm{k}=$ coeficiente de crecimiento; $\emptyset^{\prime}=$ índice de desempeño de crecimiento; $\mathrm{A}_{0.95}=$ edad máxima.

$\mathrm{L}_{\infty}=$ asymptotic length; $\mathrm{k}=$ growth coefficient; $\varnothing^{\prime}=$ growth performance index; $\mathrm{A}_{0.95}=$ max age.

de variación de $\varnothing^{\prime}$ por el método directo $(\mathrm{CV}$ $=5.26 \%$ ) indica que existe variabilidad de los parámetros de crecimiento de $H$. plumieri en diferentes regiones, mientras que por el método indirecto $(\mathrm{CV}=0.55 \%)$ no existe variación (Tabla 2).

\section{Mortalidad}

La mortalidad natural M se estimó en 1.36/ año, y la mortalidad total $Z=3.31$ año (Fig. 5), en consecuencia, se obtuvo una mortalidad por pesca de $\mathrm{F}=1.95 /$ año y la tasa de explotación $\mathrm{E}=0.59 /$ año.

\section{DISCUSIÓN}

Las longitudes encontradas en el presente trabajo para $H$. plumieri variaron entre 14 y 41 $\mathrm{cm}$, lo cual difiere con lo determinado por Potts y Manooch (2001) en el norte y sur de Carolina
(17.3-51.2 cm), Murie y Parkyn (2005) en la costa oeste de Florida (7-41 cm), Araújo y Martins (2007) en la costa central de Brasil (21.8$37.8 \mathrm{~cm}$ ), Palazón-Fernández (2007) en la isla de Margarita (18.3-38.9 cm), Constantine (2008) en la costa sur oriental de Antigua (15.9$30.9 \mathrm{~cm})$ y Shinozaki-Mendes et al. (2013a) en el estado de Ceará, Brasil (13.5-34.3 cm). Los disímiles rangos de longitudes observadas en las distintas regiones quizás estén dados por los artes de pesca utilizados en las capturas exploratorias y comerciales, como línea de mano, palangre, artes de enmalle, arrastre y nasa (Holden \& Raitt, 1975), y también por las características ecológicas de la zona de pesca. Cervigón (1993) mencionó que el mayor ejemplar observado de $H$. plumieri en el archipiélago Los Roques fue de $38.5 \mathrm{~cm}$ de longitud total, la cual es menor a la longitud total de $41 \mathrm{~cm}$ encontrada en el archipiélago Los Frailes 


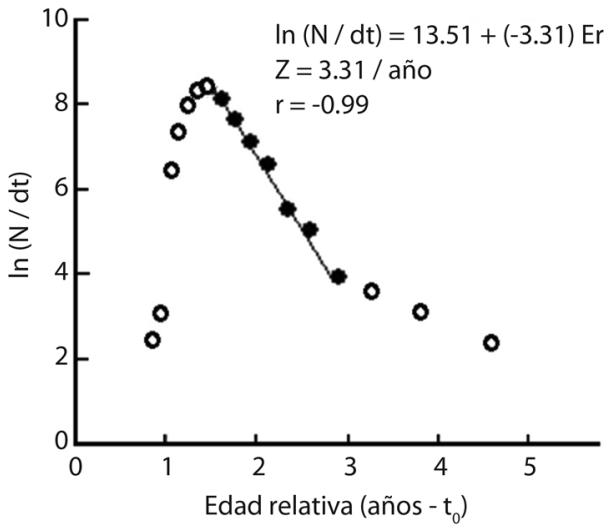

Fig. 5. Estimación de la mortalidad total (Z) de Haemulon plumieria partir del método de la curva de captura linealizada del archipiélago Los Frailes, Venezuela de enero a diciembre 2014. 1 Valores considerados en la regresión lineal.

Fig. 5. Estimation of total mortality (Z) from a linearized catch curve method of Haemulon plumieri from Los Frailes Archipelago, Venezuela between January and December 2014. 1 Values considered in the linear regression.

utilizando el mismo arte de pesca, lo que hace suponer que las longitudes máximas también están condicionadas a las zonas de pesca.

El coeficiente de regresión $b=2.99$ de la relación longitud-peso de $H$. plumieri, indica un crecimiento isométrico, es decir, existe mayor relación entre la longitud y el peso, por lo que el peso del cuerpo aumenta a un ritmo igual a la longitud (crecimiento homogéneo); diferente al crecimiento alométrico, que a medida que crecen los individuos modifican sus proporciones corporales, como es el caso ( $b=2.86)$ encontrado por Nomura (1965) en el noroeste de Brasil, Billings y Munro (1974) en Jamaica $(b=2.93)$, Potts y Manooch (2001) en el sureste de Florida $(b=2.73)$ y Murie y Parkyn (2005) en la costa central de Florida $(b$ $=2.75)$ y para la región centro-norte $(b=2.88)$. Sin embargo, Potts y Manooch (2001) reportaron en el norte y sur de Carolina $(b=3.05)$. En la isla de Margarita, Palazón-Fernández (2007) obtuvo un crecimiento alométrico diferente en adultos ( $b=2.84)$ y juveniles ( $b=3.17)$. Estos resultados indican la variabilidad de la relación longitud-peso en diferentes regiones, posiblemente, por la disparidad en la estructura de tallas de la población, debido al sexo, madurez sexual, épocas del año y alimentación (Tresierra, Culquichicón, \& Solano, 2013).

Una explicación de que no se haya presentado una progresión modal clara de la frecuencia de longitudes en el tiempo, es que $H$. plumieri es una especie tropical que se reproduce durante todo el año, con picos de máxima intensidad en febrero-abril y agosto-octubre (Palazón-Fernández, 2007). La longitud asintótica $\left(\mathrm{L}_{\infty}\right)$ determinada para $H$. plumieri presentó una considerable variación porque estuvo sujeto a las regiones y estructura de longitudes de las muestras, e.g., por el método directo (otolitos) la mayor fue de $59.1 \mathrm{~cm}$ en Carolina (Potts \& Manooch, 2001) y la menor de 28.2 $\mathrm{cm}$ en Antigua (Constantine, 2008); mientras que por el método indirecto (frecuencia de tallas) la mayor fue de $42 \mathrm{~cm}$ en Jamaica (Billings \& Munro, 1974) y la menor de 30.95 $\mathrm{cm}$ en el presente trabajo.

El coeficiente de crecimiento determinado $(\mathrm{k}=0.67 / \mathrm{año})$ indica que esta especie tiene un crecimiento moderado (Sparre \& Venema, 1997), siendo su estimación mayor al obtenido por Billings y Munro (1974) en aguas de Jamaica $(\mathrm{k}=0.37 / \mathrm{año})$ y al determinado por Potts y Manooch (2001) en el norte y sur de Carolina $(\mathrm{k}=0.08 / \mathrm{año})$. Por lo general, los peces tropicales presentan valores más altos de $\mathrm{k}$ que los peces de aguas frías por su mayor tasa metabólica (Sparre \& Venema, 1997). En el presente trabajo, la fracción adulta $>31$ $\mathrm{cm}$ no apareció regularmente en las capturas durante el periodo de muestreo, lo cual sería la causa principal de la subestimación de la longitud asintótica $\left(\mathrm{L}_{\infty}\right)$ y la sobreestimación del coeficiente de crecimiento (k). Los valores calculados del índice de crecimiento ( $\left.\varnothing^{\prime}\right)$ de H. plumieri presentan amplia diferencia, e.g., el coeficiente de variación de $\varnothing^{\prime}$ determinado por el método directo $(\mathrm{CV}=5.26 \%)$ supera el $4 \%$, lo que indica la existencia de variabilidad del patrón de crecimiento en las diferentes regiones; mientras que por el método indirecto $(\mathrm{CV}=0.55 \%)$ se demuestra lo contrario. Estas divergencias del índice de desempeño $\left(\emptyset^{\prime}\right)$ determinado por el método de los otolitos, 
puede deberse a problemas en la lectura e interpretación de los anillos de crecimiento que comprometen las estimaciones de la longitud asintótica $\left(\mathrm{L}_{\infty}\right)$ y el coeficiente de crecimiento (k). Por otro lado, Sparre y Venema (1997) señalan que los parámetros de crecimiento dentro de una misma especie, pueden variar de una población a otra, o también cohortes sucesivas pueden crecer de forma diferente según las condiciones ambientales. Algo similar ocurre con la edad máxima teórica calculada de 13 a 28 años por el método de los otolitos y de 8 a 11 años por el método de frecuencia de tallas, particularidad etaria de peces de vida larga, cuando su longitud máxima $\left(\mathrm{L}_{\infty}\right)$ es de $59.10 \mathrm{~cm}$ en el norte y sur de Carolina (Potts \& Manooch, 2001). Estos resultados difieren con el valor de 4 años obtenido en este trabajo que corresponde a una especie de vida corta.

Las tasas de mortalidad natural (M), por pesca (F) y total (Z) del presente estudio fueron mayores a las estimadas por DomínguezViveros y Ávila-Martínez (1986) en el Banco de Campeche, México ( $\mathrm{M}=1.02 / \mathrm{año}, \mathrm{F}=0.60$ / año, $Z=1.6$ /año), Potts (2000) en el norte y sur de Carolina $(\mathrm{M}=0.30 /$ año, $\mathrm{F}=0.23$ /año, $\mathrm{Z}=$ 0.53 /año) y sureste de Florida ( $M=0.30$ /año, $F$ $=0.33 /$ año, $Z=0.63 /$ año), y Araújo y Martins (2007) en la costa central de Brasil $(M=0.15 /$ año, $F=0.66 /$ año, $Z=0.21 /$ año). Sin embargo, los valores de mortalidad por pesca y total fueron menores a las determinadas por Billings y Munro (1974) en el arrecife de Port Royal, Jamaica ( $F=5.13 /$ año, $Z=6.90 / a n ̃ o)$. Sánchez y Medina (2008) indican que la expresión M es alta cuando los peces tienen crecimiento rápido y habitan ambientes cálidos. Por otra parte, las variaciones en los valores, posiblemente son causados por factores biológicos independientes de la pesca, como enfermedades, depredación, competencia, estrés del desove, inanición y vejez (Sparre \& Venema, 1997). Billings y Munro (1974) señalan que la mortalidad natural de los Hemúlidos es causada principalmente por depredación, enfermedad y vejez. La mortalidad por pesca es un parámetro importante porque puede ser controlado y regulado por el hombre; la captura o número de peces muertos por la pesca es uno de los pocos, y a veces el único dato de la población que puede ser medido y observado directamente, y puede ser relacionado posteriormente con el número de ejemplares vivos (Csirke, 1980). Es importante acotar que en las regiones costeras de Venezuela no existe una pesca dirigida al H. plumieri. En este caso, los ejemplares analizados fueron obtenidos con nasa, siendo una de las 51 especies demersales capturadas en el archipiélago Los Frailes, lo que estaría ocasionando un fuerte impacto en la pesca, cuyo efecto directo podría causar cambios en la abundancia y también en la estructura de tallas, con una reducción en las tallas máximas (Sparre \& Venema, 1997).

La tasa de explotación (E) estimada de $H$. plumieri en el presente trabajo $(\mathrm{E}=0.59 / \mathrm{año})$ fue mayor a la determinada por DomínguezViveros y Ávila-Martínez (1986) en México (E $=0.36 /$ año), Potts (2000) en el norte y sur de Carolina $(\mathrm{E}=0.43 / \mathrm{año})$ y sureste de Florida (E $=0.52 /$ año), y Araújo y Martins (2007) en Brasil $(\mathrm{E}=0.28 / \mathrm{año})$; pero menor a la estimada por Billings y Munro (1974) en Port Royal, Jamaica $(\mathrm{E}=0.74 / \mathrm{año})$. Basados en las tallas mínimas de madurez sexual de $21.3 \mathrm{~cm}$ en hembras y $27.1 \mathrm{~cm}$ en machos (Palazón-Fernández, 2007) y el alto porcentaje de juveniles (70.24 $\%$ ) en la composición de las capturas, se infiere que $H$. plumieri del archipiélago Los Frailes está siendo sobre explotada. Pauly (1983) explica que la sobrepesca puede ocurrir cuando los juveniles de las especies comerciales pasan a ser disponibles a la pesquería, es decir, los reclutas son capturados antes de alcanzar la talla necesaria. Teniendo en cuenta las características multiespecíficas de la pesquería artesanal y los antecedentes similares con Orthoristis ruber (Guerrieri, Eslava, González, \& Guevara, 2015) y Canthidermis sufflamen (Alarcón, Eslava, González, \& Guevara, 2017), se sugiere aumentar la abertura de malla y disminuir el número de nasas permitidas por pescador que faena en el archipiélago Los Frailes.

Declaración de ética: los autores declaran que todos están de acuerdo con esta publicación 
y que han hecho aportes que justifican su autoría; que no hay conflicto de interés de ningún tipo; y que han cumplido con todos los requisitos y procedimientos éticos y legales pertinentes. Todas las fuentes de financiamiento se detallan plena y claramente en la sección de agradecimientos. El respectivo documento legal firmado se encuentra en los archivos de la revista.

\section{AGRADECIMIENTOS}

Esta investigación forma parte del Proyecto "Parámetros poblacionales del corocoro margariteño Haemulon plumieri (Perciformes: Haemulidae) del archipiélago Los Frailes" Código CI 06-030603-1918-14, bajo la responsabilidad de Nora Eslava y cofinanciado por el Consejo de Investigación de la Universidad de Oriente a quienes expresamos nuestro más sinceros agradecimientos. A Juan Miguel Rodríguez, presidente de la Asociación de Pescadores Artesanales Virgen de El Carmen; así mismo, a José Arcay "Bonanza" y Onan Tineo por su apoyo logístico en la toma de datos durante el desembarco en la playa de El Tirano. A Clark Casler por la revisión y sugerencias al manuscrito.

\section{RESUMEN}

El corocoro margariteño Haemulon plumieri es muy abundante en la región nororiental de Venezuela, pero los aspectos biológicos y pesqueros han sido poco estudiados en este país. El objetivo de este estudio fue estimar el crecimiento y la mortalidad de este recurso. De enero a diciembre 2014 se realizaron muestreos mensuales y se obtuvieron 2967 ejemplares, de los cuales se registró la longitud total $(\mathrm{cm})$ y peso $(\mathrm{g})$ de cada uno. Se encontró que no existe diferencia entre sexos con respecto a la longitud ( $\left.\mathrm{t}_{\mathrm{s}}=0.093 ; \mathrm{P}>0.05\right)$, por lo que se estimó la relación longitud-peso para ambos sexos: $\mathrm{P}=1.4 \times 10^{-3} *$ $\mathrm{L}^{2.99}$, mostrando un crecimiento isométrico. Se utilizaron las rutinas del paquete FiSAT para estimar los parámetros del modelo de crecimiento individual de von Bertalanffy: $\mathrm{L}_{\infty}=30.95 \mathrm{~cm}, \mathrm{k}=0.67 /$ año, $\mathrm{P}_{\infty}=418.17 \mathrm{~g}, \mathrm{t}_{0}=-0.24$ años. Se calculó una edad máxima de 4 años. El coeficiente de variación del índice de desempeño de crecimiento $\left(\varnothing^{\prime}\right)$ para el método directo $(\mathrm{CV}=5.26 \%)$ mostró una variabilidad en el patrón de crecimiento según la región. La tasa de mortalidad natural $(\mathrm{M}=1.36 /$ año $)$ fue alta, posiblemente por causa de depredación y enfermedades, entre otros. Las tasas de mortalidad por pesca $(\mathrm{F}=1.95 /$ año $)$ y mortalidad total $(\mathrm{Z}=3.31 / \mathrm{año})$ fueron altas. La tasa de explotación (E $=0.59 /$ año) indica que es probable que $H$. plumieri esté sobreexplotado.

Palabras clave: Haemulon plumieri, crecimiento, mortalidad, archipiélago Los Frailes, Venezuela.

\section{REFERENCIAS}

Alarcón, M., Eslava, N., González, L. W., \& Guevara, F. (2017). Crecimiento y mortalidad del pez Canthidermis sufflamen (Perciformes: Balistidae) de los archipiélagos Los Frailes y Los Testigos, Venezuela. Revista de Biología Tropical, 65(3), 858-867.

Araújo, J. N., \& Martins, A. S. (2007). Age, growth and mortality of white grunt (Haemulon plumieri) from the central coast of Brazil. Scientia Marina,71(4), 793-800.

Bhattacharya, C. (1967). A simple method of resolution of a distribution into Gaussian components. Biometrics, 23, 115-123.

Billings, C. V., \& Munro, J. L. (1974). The biology, ecology and bionomics of Caribbean reef fishes: Pomadasydae (Grunts) (Part Va.). Kingston, Jamaica: University of the West Indies.

Cervigón, F. (1993). Los peces marinos de Venezuela (Vol. IV). Caracas, Venezuela: Ex Libris.

Constantine, S. (2008). Preliminary age estimates of white grunt (Haemulon plumieri) in Antiguan waters. Proceedings Gulf and Caribbean Fisheries Institute, 60, 282-286.

Csirke, J. (1980). Introducción a la dinámica de poblaciones de peces (Documento Técnico de Pesca No. 192). Roma, Italia: Organización de las Naciones Unidas para la Agricultura y la Alimentación.

Darcy, G. H. (1983). Synopsis of biological data on the grunts Haemulon aurolineatum and Haemulon plumieri (Pisces: Haemulidae) (Technical Report NMFS No. 448). Miami, United States of America: National Oceanic and Atmospheric Administration.

De Silva, J. A., \& Murphy, M. D. (2001). A summary of the states of white grunt Haemulon plumieri from the east coast of Florida (Report $\mathrm{s} / \mathrm{n}$ ). Saint Petersburg, United States of America: Florida Fish and Wildlife Conservation Commission.

Domínguez-Viveros, M., \& Ávila-Martínez, J. (1986). Diagnosis preliminar de la pesquería de chac-chi (Haemulon plumieriLacépède, 1802) del banco de Campeche en base al análisis de distribuciones de frecuencias de longitudes. Proceedings Gulf and Caribbean Fisheries Institute, 44, 747-758. 
Gayanilo Jr., F. C., Sparre, P., \& Pauly, D. (1996). FAOICLARM stock assessment tools (FiSAT). User's manual. FAO Computerized Information Series (Fisheries No. 8). Rome, Italy: United Nations Organization for Food and Agriculture.

González, L. W., Eslava, N., \& Guevara, F. (2001). Análisis de la pesca artesanal del pargo gallo (Lachnolaimus maximus) (Teleostei: Labridae) en el archipiélago Los Frailes, Venezuela. Boletín del Centro de Investigaciones Biológicas, 35(3), 272-282.

González, L. W., Eslava, N., \& Guevara, F. (2006).Catálogo de pesca artesanal del estado Nueva Esparta, Venezuela. Cumaná, Venezuela: Radoca, C. A.

González, L. W., Eslava, N., Guevara, F., Díaz, F., \& Rodríguez, J. M. (2017). Evaluación de la pesquería artesanal de El Tirano, isla de Margarita, Venezuela, durante la temporada de pesca enero-diciembre 2012. Boletín del Centro de Investigaciones Biológicas, 51(1), 43-58.

Guerrieri, A., Eslava, N., González, L. W., \& Guevara, F. (2015). Parámetros de crecimiento y mortalidad de Orthopristis ruber (Perciformes: Haemulidae) en el archipiélago Los Frailes, Venezuela. Revista de Biología Tropical, 63(1), 189-198.

Gulland, J. (1971). The fish resources of the oceans. Surrey, England: Fishing News Books Ltd.

Gulland, J., \& Holt, S. (1959). Estimation of growth parameters for data at unequal time intervals. Journal $d u$ Conseil International pour l'Exploration de la Mer $25,47-49$.

Holden, M., \& Raitt, D. (1975). Manual de Ciencia Pesquera. Parte 2. Métodos para investigar los recursos y su aplicación (Documento Técnico de Pesca No.115. Rev. 1). Roma, Italia: Organización de las Naciones Unidas para la Agricultura y la Alimentación.

Manooch, C. (1976). Age, growth, and mortality of the white grunt Haemulon plumieri Lacépède (Pisces: Pomadasydae), from North Carolina and South Carolina. Proceedings Anniversaries Conference Southeast Association Fish Wilds, Agencies, 30, 58-70.

Mota, M., \& Aragao, L. (1973). Maturação sexual do biquara, Haemulon plumieri Lacépède (Pisces: Pomadasydae). Arquivos de Ciências do Mar, 13(2), 69-76.

Munro, J., \& Pauly, D.(1983). A simple method for comparing the growth of fishes and invertebrates. Fishbyte, $1(1), 5-6$.

Murie, D., \& Parkyn, D. (2005). Age and growth of white grunt (Haemulon plumieri): a comparison of two populations along the west coast of Florida. Bulletin of Marine Science, 76(1), 73-93.
Murphy, M., Murie, D., \& Muller, R. (1999). Stock assessment of white grunt from the west coast of Florida (Report $\mathrm{s} / \mathrm{n}$ ). Florida, United States of America: Florida Fish and Wildlife Conservation Commission.

Nomura, H. (1965). Length-weight tables of some fish species from northeastern Brazil. Arquivos de Estação de Biologia Marinha da Universidade Federal do Ceará, 5(2), 103-105.

Palazón-Fernández, J. (2007). Reproduction of the white grunt, Haemulon plumieri (Lacépède, 1802) (Pisces: Haemulidae) from Margarita Island, Venezuela. Scientia Marina, 71(3), 429-440.

Pauly, D. (1979). Theory and Management of tropical multispecies stocks: a review, with emphasis on the Southeast Asian demersal fisheries. International Center for Living Aquatic Resources Management Studies and Review, 1, 1-35.

Pauly, D. (1980). On the interrelationships between natural mortality, growth parameters and mean environmental temperature in 175 fish stocks. International Council for the Exploration of the Sea, 39(2), 175-192.

Pauly, D. (1983). Algunos métodos simples para la evaluación de recursos pesqueros tropicales (Documento Técnico de Pesca No. 234). Roma, Italia: FAO.

Pauly, D., \& David, N. (1981). ELEFAN I, a BASIC program for the objective extraction of growth parameters from length-frequency data. Meeresforschung, 28(4), 205-211.

Potts, J. (2000). Population assessment of two stocks of white grunt Haemulon plumieri from the southeastern coast of the United States (NOAA Technical Memorandum NMFS-SEFSC No. 442). Miami, United States of America: National Oceanic and Atmospheric Administration.

Potts, J., \& Manooch, C. (2001). Differences in the age and growth of white grunt (Haemulon plumieri) from North Carolina and South Carolina compared with southeast Florida. Bulletin of Marine Science, $68(1), 1-12$

Powell, D. (1979). Estimation of mortality and growth parameters for the length frequency of a catch. Rapport procès-V Réunion Conseil International pour L'Exploitation de la Mer, 175, 167-169.

Ricker, W. (1975). Computation and interpretation of biological statistic of fish population. Department of Environmental Fisheries and Marine Service. Bulletin of Fisheries Research Board of Canada, 191, 382.

Sadovy, Y., Figuerola, M., \& Román, A. (1989). The age and growth of the red hind Epinephelus guttatus and the white grunt Haemulon plumieri in Puerto Rico and the U.S. Virgin Islands (Final Report $\mathrm{s} / \mathrm{n}$ ). San Juan, Puerto Rico: Caribbean Fishery Management Council. 
Sánchez, O., \& Medina, A. (2008). Análisis de la pesquería de boquinete, Lachnolaimus maximus, en la Isla de Holbox, Quintana Roo, México. Proceedings Gulf and Caribbean Fisheries Institute, 60, 287-296.

Shinozaki-Mendes, R., Santander, J., Silva, J., \& Hazin, F. (2013a). Gonad maturation of Haemulon plumieri (Teleostei: Haemulidae) in Ceará State, Northeastern Brazil. Brazilian Journal of Biology, 73(2), 383-390.

Shinozaki-Mendes, R., Santander, J., Silva, J., \& Hazin, F. (2013b). Reproductive biology of Haemulon plumieri (Teleostei: Haemulidae) in Ceará State, Northeastern Brazil. Brazilian Journal of Biology, 73(2), 391-396.

Sparre, P., \& Venema, S. (1997). Introducción a la evaluación de recursos pesqueros tropicales (Documento Técnico de Pesca No. 306, Parte 1 Rev. 2). Roma, Italia: Organización de las Naciones Unidas para la Agricultura y la Alimentación.

Taylor, C. (1958). Cod growth and temperature. Journal du Conseil International pour l'Exploration de la Mer, 23, 366-370.
Treinen-Crespo, C., Villegas-Hernández, H., Guillén-Hernández, S., Ruiz-Zárate, M., \& González-Salas, C. (2012). Análisis de la forma del otolito como herramienta para la discriminación poblacional del stock del ronco (Haemulon plumieri) en la costa norte de la Península de Yucatán, México. Revista Ciencias Marinas y Costeras, 4, 157-168.

Tresierra, A., Culquichicón, Z., \& Solano, A. (2013). Métodos para la gestión de los recursos pesqueros ( $1^{\mathrm{a}}$ ed.). Trujillo, Perú: Industria Gráfica ABC SAC.

von Bertalanffy, L. (1938). A quantitative theory of organic growth. Human Biology, 10(2), 181-213.

Walpole, R., Myers, R., Myers, S., \& Ye, K. (2007). Probability \& statistics for engineer \& scientists. New Jersey, United States of America: Prentice Hall.

Wetherall, J. (1986). A new method for estimating growth and mortality parameters from length-frequency data. Fishbyte, 4, 12-14.

Zar, J. (2009). Biostatistical analysis. New Jersey, United States of America: Prentice Hall. 